\title{
Facilitation of lethal ventricular arrhythmias by therapeutic digoxin in conscious post infarction dogs
}

\begin{abstract}
The proarrhythmic potential of digoxin, administered in a therapeutic dosage regimen, was evaluated in conscious dogs in the subacute phase of myocardial infarction. In this evaluation, digoxin $(0.0125 \mathrm{mg} / \mathrm{kg}$ /day intravenously) or vehicle were administered to conscious dogs for periods of 5 to 7 days, commencing 4 to 5 days after anterior myocardial infarction. Before treatment, programmed ventricular stimulation failed to initiate ventricular tachycardia in 26 post infarction dogs. After treatment, programmed stimulation initiated ventricular tachyarrhythmias in only 1 of 13 digoxin-treated dogs $(1.36 \pm 0.17 \mathrm{ng} / \mathrm{ml}$ serum digoxin) and in 0 of 13 vehicle-treated dogs. However, the incidences of early ventricular fibrilatlion (4 of 10 digoxin vs 0 of 12 vehicle; $p<0.05$ ) and of 24-hour mortality ( 6 of 10 digoxin vs 2 of 12 vehicle; $p<0.05$ ) occurring in response to the development of posterolateral ischemia in the presence of previous anterior myocardial infarction was significantly greater in digoxin-treated $(1.47 \pm 0.19 \mathrm{ng} / \mathrm{ml}$ serum digoxin) than in vehicle-treated animals. These findings suggest an enhanced susceptibility toward the development of ischemia-related lethal arrhythmias in the presence of therapeutic digoxin serum concentrations early after myocardial infarction, which is not predicted by programmed ventricular stimulation testing. (AM HEART J 111:883, 1986.)
\end{abstract}

Joseph J. Lynch, Ph.D., Daniel G. Montgomery, B.S., and Benedict R. Lucchesi, Ph.D., M.D. Ann Arbor, Mich.

Considerable uncertainty surrounds the use of digitalis in patients with ischemic myocardial injury., Several large nonrandomized clinical studies have attempted to evaluate the potential deleterious effects of digitalis therapy on survival after acute myocardial infarction, but have yielded conflicting and equivocal results. ${ }^{3-7}$ Experimental studies conducted to date suggest an enhanced myocardial sensitivity to the arrhythmogenic actions of digitalis preparations in the settings of myocardial ische$\mathrm{mia}^{8-10}$ and/or myocardial infarction. ${ }^{8,9,11-15}$ However, all previous experimental studies invariably have employed the rapid administration of toxic concentrations of digitalis preparations in assessing myocardial sensitivity, ${ }^{8-15}$ thereby limiting the relevance of conclusions drawn from such studies to the clinical situation.

From the Department of Pharmacology, The University of Michigan Medical School.

This study was supported by the National Institutes of Health, Heart, Lung, and Blood Institute, Grant No. HL-05806-24. Dr. Lynch is a fellow of the American Heart Association of Michigan.

Received for publication June 13, 1985; revision received Sept. 3, 1985; accepted Oct. 7, 1985.

Reprint requests: Benedict R. Lucchesi, Ph.D., M.D., Dept. of Pharmacology, M6322 Medical Science Bldg. I, The University of Michigan Medical School, Ann Arbor, MI 48109.
Previously, ${ }^{16}$ this laboratory has described a conscious canine model for the study of sudden coronary death precipitated by the development of ischemia at a site distant from an area of previous myocardial infarction. More recent studies have demonstrated a predictive relationship between susceptibility to the initiation of ventricular tachyarrhythmias by programmed ventricular stimulation and vulnerability to the development of lethal ventricular arrhythmias in response to a subsequent ischemic episode in this conscious post infarction dog model. ${ }^{17}$ This relationship permits the identification of post infarction animals at low risk to the development of ischemia-related malignant ventricular arrhythmias via prior programmed ventricular stimulation testing. ${ }^{17}$ In the present investigation, post infarction dogs which were determined to be at a low risk for the development of ischemia-induced lethal arrhythmias were employed to evaluate the arrhythmogenic potential of the digitalis glycoside, digoxin, administered in a therapeutic dosing regimen.

\section{METHODS}

Surgical preparation. Male mongrel dogs (14.0 to 19.0 $\mathrm{kg}$ ) were anesthetized with intravenous sodium pentobarbital, $30 \mathrm{mg} / \mathrm{kg}$. The dogs were ventilated with room air 


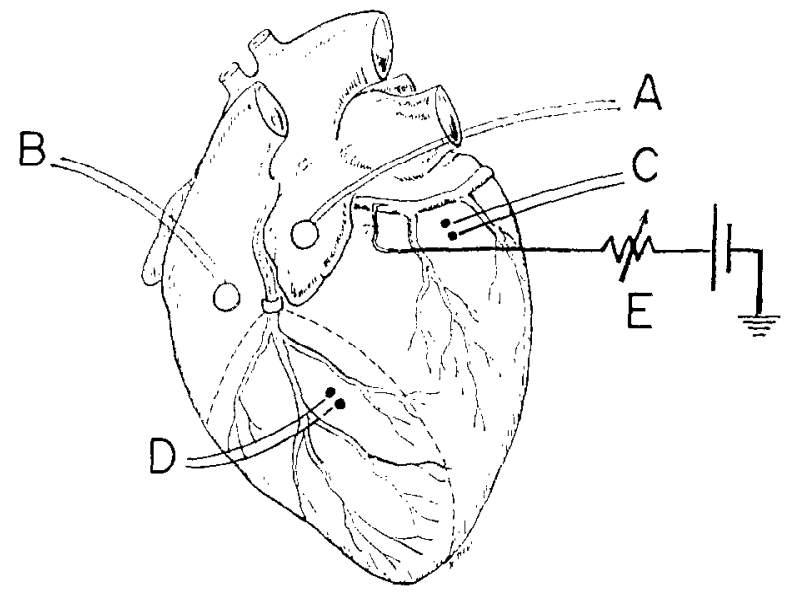

Fig. 1. Conscious post infarction canine model. On the day of surgical preparation, anterior myocardial ischemic injury (demarcated by the broken line) was produced by a 2 -hour occlusion of the left anterior descending coronary artery, followed by reperfusion through a critical stenosis. An epicardial bipolar electrode $(A)$ was sewn onto the left atrial appendage for atrial pacing, and a plunge bipolar electrode $(B)$ was inserted into the interventricular septum, adjacent to the site of occlusion, for the determination of ventricular excitation threshold and refractory period, as well as for the delivery of programmed ventricular extrastimuli. Plunge bipolar electrodes were inserted into normal, noninjured myocardium (C) and into potentially infarcted myocardium $(D)$ for the recording of local activation, and for the construction of ventricular strength-interval curves. The bared tip of an insulated silver wire $(E)$ was inserted into the lumen of the left circumflex coronary artery, and the wire was sutured onto the surface of the heart.

with the use of a cuffed endotracheal tube and a Harvard respirator. By the use of the aseptic technique, the left external jugular vein and left common carotid artery were isolated, and cannulas were inserted. A left thoracotomy was performed between the fourth and fifth ribs. The pericardium was opened and the heart was suspended in a pericardial cradle. The left anterior descending coronary artery was isolated at the tip of the left atrial appendage, and the left circumflex coronary artery was isolated approximately $1 \mathrm{~cm}$ from its origin. A 19- or 20-gauge hypodermic needle was placed parallel to the left anterior descending coronary artery and a suture was passed around both the vessel and the needle. The suture was tied securely and the needle was withdrawn, producing a critical stenosis. The artery was then occluded using a snare formed from a loop of silicone rubber tubing passed through a polyethylene tube. Blood flow through the left anterior descending coronary artery was restored after 2 hours.

An epicardial bipolar electrode ( $1 \mathrm{~mm}$ diameter silver electrodes embedded $3 \mathrm{~mm}$ apart in acrylic) was sutured to the left atrial appendage for atrial pacing. A bipolar plunge electrode (25-gauge insulated stainless steel wire, 5 $\mathrm{mm}$ in length, $2 \mathrm{~mm}$ apart) was sutured into the interven- tricular septum adjacent to the right ventricular outflow tract for the determination of ventricular excitation threshold, ventricular refractory period, and for the introduction of ventricular extrastimuli during programmed stimulation. Two short ( 2 to $3 \mathrm{~mm}$ ) stainless steel bipolar plunge electrodes were used for the measurement of ventricular activation times and for the construction of ventricular strength-interval curves. One short bipolar electrode was implanted in the distribution of the left anterior descending coronary artery distal to the site of occlusion (IZ, infarct zone), while a second electrode was implanted in the left circumflex coronary artery distribution (NZ, normal zone). A $3 \mathrm{~mm}$ section of bared insulated 30-gauge silver wire was inserted through the wall and to the lumen of the left circumflex coronary artery and was sutured in place on the surface of the heart. Silver disc electrodes were implanted subcutaneously. The surgical incision was closed and the animals were allowed to recover from surgical anesthesia. The animals received ampicillin, $10 \mathrm{mg} / \mathrm{kg}$ subcutaneously twice daily, during their post infarction convalescence. The essential cxperimental features of this preparation are illustrated in Fig. 1.

Experimental protocol. Post infarction dogs were returned to the laboratory 4 to 5 days after surgical preparation. Electrophysiologic testing and programmed ventricular stimulation were performed while the animals were conscious, unsedated, and resting comfortably in a sling. Only post infarction dogs that were not susceptible to the initiation of ventricular tachycardia during initial programmed stimulation testing were entered into the present investigation. Previous work in this laboratory has shown that post infarction dogs that are resistant to the induction of ventricular tachycardia by programmed stimulation are at "low risk" toward the development of lethal ventricular arrhythmias in response to the subsequent development of posterolateral ischemia (i.e., ischemia at a site distant from the area of previous myocardial infarction). ${ }^{17}$

After initial electrophysiologic testing, post infarction dogs were randomized to treatment with either digoxin ( $\mathrm{n}=13,0.0125 \mathrm{mg} / \mathrm{kg} /$ day intravenous Lanoxin, administered in the afternoon as a single infusion via the jugular vein, for 5 to 7 consecutive days) or with vehicle $(n=13$, digoxin diluent in matching volume, administered as a single afternoon infusion, for 5 to 7 consecutive days). Daily morning blood samples were obtained by peripheral venipuncture from the first six digoxin-treated animals for the characterization of the serum digoxin-time course profile. Morning venous blood samples were obtained from all digoxin and vehicle-treated animals for the determination of serum potassium and digoxin concentrations on the day of repeat electrophysiologic testing.

After the appropriate treatment periods, digoxin- and vehicle-treated dogs were subjected to repeat electrophysiologic study and programmed ventricular stimulation in the conscious, unsedated state. Immediately thereafter, digoxin-treated $(n=10)$ and vehicle-treated $(n=12)$ dogs were entered into the protocol for left circumflex (LCX) 
coronary artery intimal injury. Technical failures associated with the latter protocol (fractured or improperly positioned LCX wire, $\mathrm{n}=3$; two digoxin- and one vehicletreated) and lethal responses to programmed stimulation $(n=1)$ account for the reduction in the number of animals entered into the protocol for LCX coronary artery injury.

Electrophysiologic studies and programmed electrical stimulation. Animals were studied while they were conscious, unsedated, and resting comfortably in a sling. ECG intervals and electrophysiologic parameters were determined immediately before programmed stimulation testing. ECG intervals were measured during sinus rhythm. A corrected QT interval $\left(\mathrm{QT}_{c}=\mathrm{QT}\right.$ in $\mathrm{msec} /(\mathrm{R}-\mathrm{R}$ in sec $)^{1 / 2}$ ) was determined during sinus rhythm, while a paced QT interval was determined during atrial pacing $(2.5 \mathrm{~Hz})$. Ventricular excitation thresholds, refractory periods, and activation times were determined during atrial pacing $(2.5 \mathrm{~Hz})$. 'I'he ventricular excitation threshold was defined as the minimum voltage required to produce a conducted ventricular beat at a stimulus duration of $4 \mathrm{msec}$ delivered $300 \mathrm{msec}$ after the $R$ wave of the lead II ECG. The ventricular refractory period was the longest $\mathrm{R}$-to-stimulus interval at which a $2 \mathrm{x}$ threshold stimulus ( 4 msec duration) failed to produce a conducted ventricular beat. Normal zone and infarct zone ventricular activation times, the intervals between the $Q$ wave of the lead II ECG, and the largest deflection of the local ventricular electrograms ( $\mathrm{Q}-\mathrm{EG}$ ), were measured on a Tektronix model 5111 oscilloscope. Ventricular strengthinterval curves were constructed in normal and infarcted myocardium by a modification of a previously reported method. ${ }^{18}$ An extrastimulus $\left(\mathrm{S}_{2}\right)$ is introduced in late diastole at a minimum milliamperage for eliciting a ventricular response $\left(V_{2}\right)$ during a basic $V_{1}-V_{1}$ drive cycle length of $400 \mathrm{msec}$ (heart rate of $150 \mathrm{bpm}$ ) in the conscious dog. The current for the drive beats is held constant throughout the determination at twice the minimum diastolic threshold for excitability. The coupling interval of $S_{2}$ is then decreased incrementally until $S_{2}$ fails to elicit a $V_{2}$. When $S_{2}$ fails to elicit a $V_{2}$, the milliamperage of $S_{2}$ is increased until a $V_{2}$ is elicited, and the coupling interval is decreased until $S_{2}$ again fails to elicit a $V_{2}$. This sequence is repeated until a ventricular effective refractory period (ERP) is reached at a maximum current of $4 \mathrm{~mA}$. The ERP, therefore, is defined as the longest $S_{1}-S_{2}$ interval that fails to elicit $V_{2}$ at $4 \mathrm{~mA}$. The relative refractory period (RRP) is defined as the longest coupling interval along the strength-interval curve at which the current required to evoke a response $\left(\mathrm{V}_{2}\right)$ increases above the diastolic excitability threshold (ET) by greater than 0.025 $\mathrm{mA}$ for a $1 \mathrm{msec}$ change in coupling interval. ${ }^{18}$

During the programmed electrical stimulation protocol, premature ventricular stimuli $(4 \mathrm{msec}$ duration, $2 \times$ threshold) were introduced into the interventricular septum using a Grass model S-88 stimulator and a Grass model SIU-5 stimulus isolation unit. Single $\left(\mathrm{S}_{2}\right)$, double $\left(S_{2}-S_{3}\right)$, and then triple $\left(S_{2}-S_{3}-S_{4}\right)$ premature ventricular stimuli were introduced. Single ventricular extrastimuli

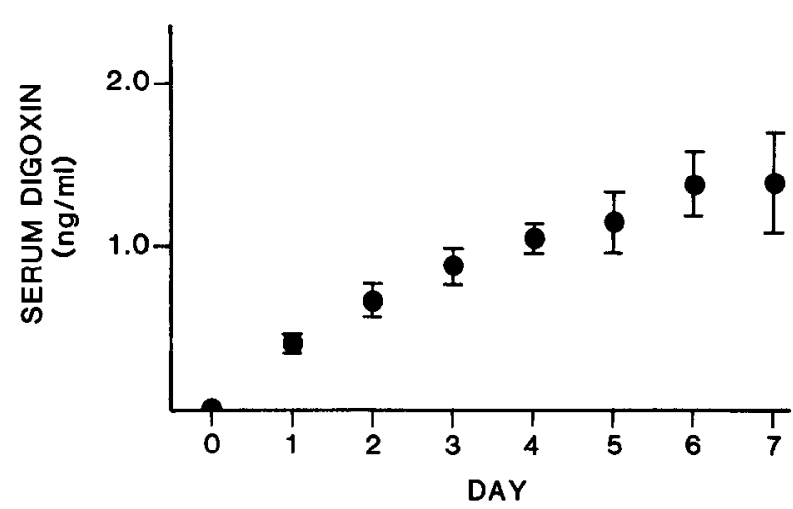

Fig. 2. Serum digoxin concentrations were determined daily for the first six digoxin-treated post infarction dogs $(0.0125 \mathrm{mg} / \mathrm{kg} / \mathrm{day}$ digoxin $)$. Serum digoxin concentrations (mean \pm S.E.M.) are plotted as a function of treatment day during the treatment period.

were introduced during atrial pacing at $S_{1}-S_{2}$ coupling intervals decreasing from $350 \mathrm{msec}$ until ventricular refractoriness occurred. Thereafter, double and triple ventricular extrastimuli were introduced during sinus rhythm at $S_{2}-S_{3}$ and $S_{2}-S_{3}-S_{4}$ coupling intervals of 182,167 , $154,143,133$, and 125 msec. Previous work has shown that this method fails to produce ventricular dysrhythmias in sham-operated animals without previous myocardial ischemic injury. ${ }^{16}$

Ischemia at a site remote from previous myocardial infarction: Left circumflex intimal stimulation and injury. After the completion of post treatment programmed ventricular stimulation testing, an anodal direct current of $150 \mu \mathrm{A}$ was applied to the intimal surface of the left circumflex coronary artery via the previously inserted silver wire electrode. The lead II ECG was recorded directly onto a Grass polygraph or was recorded, at preset intervals, by a programmable cardiocassette recorder.

Upon completion of the experiment at 24 hours of electrical stimulation or upon the development of ventricular fibrillation, the heart was excised, and thrombus mass within the left circumflex coronary artery was determined after removal by careful dissection. The heart was cut into $1 \mathrm{~cm}$ thick transverse sections which were placed in $0.5 \%$ triphenyltetrazlium chloride in $0.01 \mathrm{M}$ phosphate buffer (pH 7.4). Infarct size was quantitated gravimetrically with the aid of the differential histochemical reaction. ${ }^{16}$

Determination of serum potassium and digoxin concentrations. Blood samples for the determination of serum digoxin and potassium concentrations were obtained by venipuncture 16 hours after the preceding administration of digoxin. Serum potassium concentrations were determined by flame photometry. Serum digoxin concentrations were determined by means of radioimmunoassay technique in the Ligand Assay Laboratory of the University of Michigan Medical School, with the use of sheep antidigoxin antiserum.

Statistical analysis. For all evaluations, data are expressed as the mean \pm S.E.M. Within a given treatment 
Table I. Electrocardiographic and electrophysiologic responses*

\begin{tabular}{|c|c|c|c|c|}
\hline \multirow[b]{2}{*}{ Parameter } & \multicolumn{2}{|c|}{$\begin{array}{l}\text { Digoxin } \\
(n=13)\end{array}$} & \multicolumn{2}{|c|}{$\begin{array}{l}\text { Vehicle } \\
(n=13)\end{array}$} \\
\hline & Before & After & Before & After \\
\hline Heart rate $(\mathrm{bpm})$ & $109 \pm 5$ & $115 \pm 7$ & $100 \pm 7$ & $102 \perp 6$ \\
\hline Mean arterial pressure $(\mathrm{mm} \mathrm{Hg})$ & $79 \pm 4$ & $79 \pm 7$ & $78 \pm 3$ & $84 \pm 5$ \\
\hline PR interval (msec) & $118 \pm 3$ & $120 \pm 4$ & $123 \pm 4$ & $125 \pm 5$ \\
\hline QRS interval (msec) & $59 \pm 1$ & $58 \pm 2$ & $56 \pm 1$ & $55 \pm 1$ \\
\hline $\mathrm{QT}_{\mathrm{c}}$ interval $(\mathrm{msec}) /(\mathrm{sec})^{1 / 3}$ & $285 \pm 8$ & $270 \pm 5$ & $265 \pm 6$ & $274 \pm 6$ \\
\hline Paced QT interval (msec) & $205 \pm 7$ & $189 \pm 5+$ & $198 \pm 7$ & $202 \pm 6$ \\
\hline Ventricular excitation threshold voltage $(\mathrm{V})^{*}$ & $2.2 \pm 0.2$ & $2.5 \pm 0.3$ & $2.7 \pm 0.6$ & $3.5 \pm 0.8$ \\
\hline Ventricular refractory period (msec) ${ }^{*}$ & $163 \pm 6$ & $136 \pm 6 \ddagger$ & $159 \pm 7$ & $140 \pm 5 \dagger$ \\
\hline
\end{tabular}

${ }^{*}$ Electrophysiologic parameters determined at the right ventricular outflow tract site during programmed ventricular stimulation. $\dagger p<0.05, \ddagger p<0.01$ before $v s$ after within the treatment group.

Table II. Electrophysiologic responses in normal noninfarcted and infarcted zones of myocardium

\begin{tabular}{|c|c|c|c|c|}
\hline \multirow[b]{2}{*}{ Site and parameter } & \multicolumn{2}{|c|}{ Digoxin } & \multicolumn{2}{|c|}{ Vehicle } \\
\hline & Before & After & Before & After \\
\hline \multicolumn{5}{|l|}{ "Normal zone"* } \\
\hline Excitation threshold (mA) & $0.36 \pm 0.03$ & $0.56 \pm 0.15$ & $0.31 \pm 0.07$ & $0.53 \pm 0.22$ \\
\hline Relative refractory period (msec) & $166 \pm 7$ & $146 \pm 3 \dagger$ & $167 \pm 7$ & $151 \pm 6$ \\
\hline Efrective refractory period (msec) & $141 \perp 7$ & $125 \pm 3 \dagger$ & $139 \pm 5$ & $126 \pm 12$ \\
\hline Activation time (msec) & $20.5 \pm 1.6$ & $22.6 \pm 1.9$ & $20.7 \pm 2.0$ & $19.8 \pm 2.0$ \\
\hline \multicolumn{5}{|l|}{ "Infarct zone"* } \\
\hline Excitation threshold (mA) & $0.48 \pm 0.07$ & $0.90 \pm 0.26$ & $0.30 \pm 0.04$ & $0.49 \pm 0.09$ \\
\hline Relative refractory period (msec) & $182 \pm 9$ & $135 \pm 4 \hbar \S \|$ & $179 \pm 7$ & $153 \pm 4 \dagger$ \\
\hline Effective refractory period (msec) & $152 \pm 7$ & $117 \pm 5 \ddagger$ & $142 \pm 5$ & $124 \pm 2 \dagger$ \\
\hline Activation time (msec) & $24.2 \pm 2.2$ & $22.1 \pm 2.3$ & $20.5 \pm 1.6$ & $19.2 \pm 1.9$ \\
\hline
\end{tabular}

* For all normal zone parametcrs, $\mathrm{n}=13$; infarct zone values for excitation threshold, relative and effective refractory periods are $\mathrm{n}=9$ and $\mathrm{n}=8$ for the digoxin and vehicle treatment groups, respectively. The reduction in $n$ in the infarct zone was due to the inability to construct strength-interval curves in electrically inexcitable tissue in several animals.

$\mp p<0.05, \ddagger p<0.01$ before us alter within the treatment group and within the same zone of myocardium.

$\$ p<0.01$ infaret zone post parameter vs paired normal zone post parameter within the same treatment group.

$\| p<0.05$ digoxin post treatment parameter os analogous vehicle post treatment parameter within the same zone of myocardium.

group, pre- $v s$ post treatment comparisons and normal zone us infarct zone comparisons were made using a two-tailed paired Student's $t$ test. Differences between the two treatment groups were analyzed by a two-tailed unpaired $t$ test or by a Fisher's exact test, when appropriate. For all comparisons, a $p$ value of less than 0.05 was the criterion for statistical significance.

\section{RESULTS}

Twenty-six conscious post infarction dogs were entered into the present study. These animals were randomized to treatment with digoxin $(n=13$; $0.0125 \mathrm{mg} / \mathrm{kg} /$ day intravenously for a mean treatment period of $5.9 \pm 0.4$ days), or with vehicle ( $n=13$; digoxin diluent administered in matching volume for a mean treatment period of $5.3 \pm 0.4$ days). The pre- and post treatment responses of the post infarction dogs to electrophysiologic testing and programmed ventricular stimulation, as well as the responses of the treated animals to the subse- quent development of acute posterolateral ischemia, are presented below.

Serum digoxin-Time course profile. Daily determinations of serum digoxin concentrations were performed for the first six digoxin-treated dogs completing a 7-day treatment period. Serum samples for the digoxin assay were obtained by peripheral venipuncture 16 hours after the preceding administration of digoxin. After 4 to 7 days of treatment, serum digoxin concentrations ranging from 0.80 to 2.31 $\mathrm{ng} / \mathrm{ml}$ were achieved in these animals. Mean serum digoxin concentrations for treatment days 4 through 7 for this group ranged from $1.12 \pm 0.30$ to $1.40 \pm$ $0.32 \mathrm{ng} / \mathrm{ml}$ (Fig. 2). All digoxin-treated dogs in this study were administered intravenous digoxin for treatment periods of 5 to 7 days before repeat programmed ventricular stimulation and electrophysiologic testing.

Programmed electrical stimulation. Twenty-six post infarction dogs were subjected to repeat pro- 
Table III. Response to ischemia at a site remote from previous myocardial infarction

\begin{tabular}{|c|c|c|}
\hline Parameter & $\begin{array}{l}\text { Digoxin } \\
(n=10)\end{array}$ & $\begin{array}{c}\text { Vehicle } \\
(n=12)\end{array}$ \\
\hline Time to ischemia (min) & $298 \pm 33$ & $219 \pm 35$ \\
\hline $\begin{array}{l}\text { Increase in heart rate } \\
\text { upon ST segment } \\
\text { change ( } \% \text { of } \\
\text { pre-ischemic rate) }\end{array}$ & $+28 \pm 7$ & $+39 \pm 7$ \\
\hline $\begin{array}{l}\text { Incidence of "sudden" } \dagger \\
\text { ventricular fibrillation } \\
\text { upon ischemia }\end{array}$ & $4 / 10^{*}$ & $0 / 12$ \\
\hline 24-hour mortality & $6 / 10^{*}$ & $2 / 12$ \\
\hline \multicolumn{3}{|l|}{$\begin{array}{l}\text { Infarct size }(\% \text { of left } \\
\text { ventricle })\end{array}$} \\
\hline Left anterior descending & $6.6 \pm 1.4$ & $6.3 \pm 1.8$ \\
\hline Left circumflex & $\begin{array}{c}18.3 \pm 3.2 \\
(\mathrm{n}=6)\end{array}$ & $21.5 \pm 2.3$ \\
\hline Thrombus mass & $12.2 \pm .9$ & $13.4 \pm 3.2$ \\
\hline
\end{tabular}

${ }^{*} p<0.05$ digoxin $u s$ vehicle treatment group.

$\dagger$ Incidence of ventricular fibrillation within 60 minutes of ischemia.

grammed ventricular stimulation in the conscious unsedated state after digoxin $(n=13)$ or vehicle $(n=13)$ treatment. Before treatment, all dogs had been determined noninducible during programmed stimulation testing. At the time of retesting, animals in the digoxin-treated group had a mean serum digoxin concentration of $1.36 \pm 0.17 \mathrm{ng} / \mathrm{ml}$, and all dogs in both groups appeared healthy and displayed no spontaneous ventricular ectopy. Repeat programmed ventricular stimulation failed to initiate nonsustained or sustained ventricular tachycardia in 12 of 13 digoxin-treated and in 13 of 13 vehicletreated animals. One previously noninducible digoxin-treated dog responded to repeat programmed ventricular stimulation with a rapid polymorphic ventricular tachycardia that degenerated into ventricular fibrillation, and was not resuscitated. The sizes of the underlying anterior myocardial infarctions did not vary significantly between the digoxintreated $(7.1 \pm 1.4 \%$ of left ventricle) and vehicletreated $(6.3 \pm 1.6 \%$ of left ventricle) animals subjected to repeat programmed stimulation.

Electrophysiologic responses. Table I summarizes the pre- and post treatment values for ECG intervals, sinus heart rate, mean arterial pressure, and for ventricular excitation threshold voltage and refractory period, determined at the right ventricular outflow tract (RVOT) site during programmed ventricular stimulation. For both treatment groups, post treatment RVOT refractory periods were significantly less than their respective pretreatment values, with the reduction in refractoriness in the digoxin treatment group being greater in magnitude

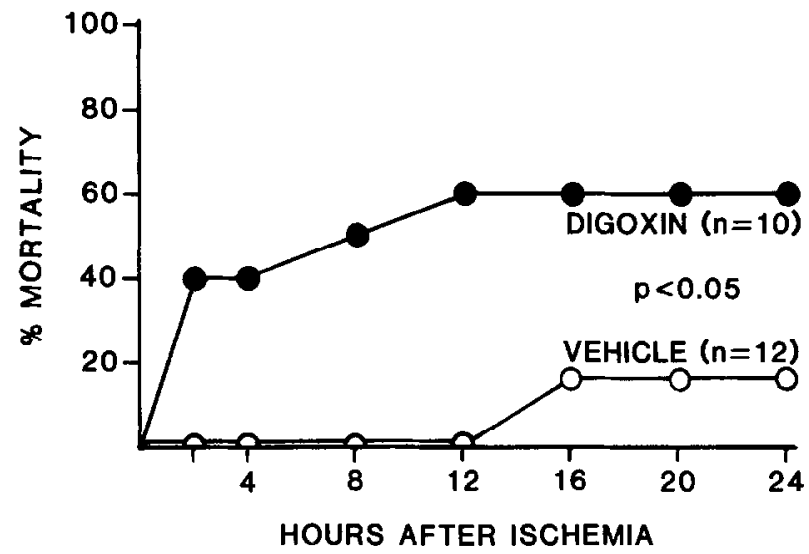

Fig. 3. Mortality rates of digoxin- and vehicle-pretreated post infarction dogs after the development of posterolateral ischemia following left circumflex intimal injury.

than that observed in the vehicle treatment group (Table I). Digoxin administration to post infarction dogs resulted in a significant reduction in the $Q^{\prime} T$ interval, while ECG intervals were unaltered in the vehicle group (Table I).

Pre- and post treatment values for ventricular activation time, ET, RRP, and ERP, determined in NZs and IZs of myocardium, are presented in Table II. For both the digoxin and vehicle treatment groups, the NZ and IZ post treatment values for RRP and ERP were less than their appropriate pretreatment values. However, the reductions in relative and effective refractoriness for both noninfarcted and infarcted myocardium were consistently greater in magnitude for the digoxin treatment group than for the vehicle treatment group (Table II). The most striking electrophysiological alteration observed in this evaluation was the reduction in the RRP value for infarcted myocardium in the digoxin treatment group. After digoxin administration, the $R R P$ value in infarcted myocardium was significantly less than the post treatment RRP value in noninfarcted myocardium within the digoxin treatment group, and additionally was significantly less than the corresponding post treatment RRP value in infarcted myocardium in the vehicle treatment group (Table II).

Response to ischemia at a site remote from previous myocardial infarction. Immediately after post treatment programmed ventricular stimulation, an anodal current of $150 \mu \mathrm{A}$ was applied to the intimal surface of the left circumflex coronary artery in each of 10 digoxin-treated and 12 vehicle-treated post infarction dogs. At the time of this procedure, the animals in the digoxin-treated group had a mean serum digoxin concentration of $1.47 \pm 0.19 \mathrm{ng} / \mathrm{ml}$. Serum potassium concentrations of digoxin-treated 


\section{LCX INTIMAL STIMULATION (150 UA ANODAL)- DIGOXIN $0.0125 \mathrm{MG} / \mathrm{KG} / \mathrm{DAY}$}
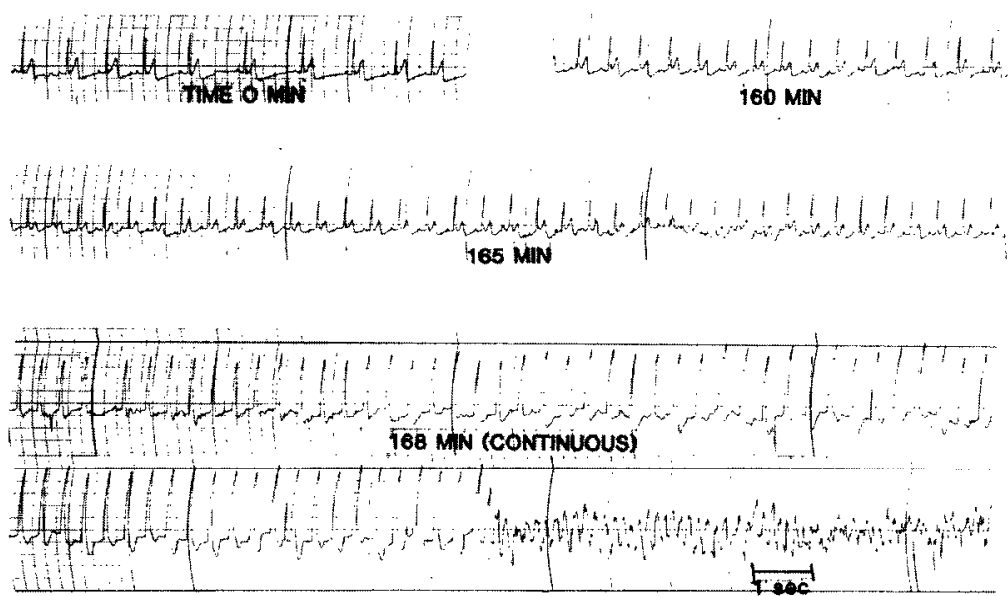

Fig. 4. Ventricular fibrillation in a digoxin-pretreated conscious dog in response to the development of posterolateral ischemia in the presence of anterior myocardial infarction. An anodal current of $150 \mu \mathrm{A}$ was applied to the intimal surface of the left circumflex $(L C X)$ coronary artery at time 0 . In this experiment, ECG alterations reflecting acute ischemia (widened QRS with increasing $R$ wave amplitude, reflex tachycardia) was observed at 257 minutes of current, and were followed rapidly by the development of ventricular fibrillation. Depicted ECG is lead II.

$(3.72 \pm 0.09 \mathrm{mEq} / \mathrm{L})$ and vehicle-treated $(3.66 \pm$ $0.15 \mathrm{mEq} / \mathrm{L}$ ) dogs entering this phase of the evaluation were not significantly different. The responses of the two treatment groups to left circumflex intimal stimulation are summarized in Table III.

The responses of digoxin- and vehicle-pretreated dogs to posterolateral ischemia in the presence of a previous anterior infarction are compared graphically in the mortality curves depicted in Fig. 3. Within 60 minutes of the onset of ischemia, 4 of 10 digoxintreated dogs vs 0 of 12 vehicle-treated dogs had developed ventricular fibrillation $(40 \%$ digoxin vs $0 \%$ vehicle; $p<0.05$ ). The mean time to the development of ventricular fibrillation after the onset of ischemia in these four digoxin-treated dogs was $5.3 \pm 0.4$ minutes, and the serum digoxin concentrations for these four animals ranged from 0.82 to 1.32 $\mathrm{ng} / \mathrm{ml}$. The incidence of arrhythmic fatalities at 24 hours after the development of posterolateral ischemia was 6 of 10 in the digoxin treatment group and 2 of 12 in the vehicle treatment group (60\% digoxin $v$ s $17 \%$ vehicle; $p<0.05$ ), with two "late" arrhythmic deaths occurring in each of the two groups. Fig. 4 illustrates the development of ventricular fibrillation shortly after the development of posterolateral ischemia in a digoxin-treated post infarction dog.

\section{DISCUSSION}

Previous clinical studies. The use of digitalis after myocardial infarction remains controversial, due primarily to an uncertainty regarding the arrhythmogenic potential of digitalis in the setting of ischemic myocardial injury. ${ }^{1,2}$ Initial clinical investigations have not provided unequivocal evidence of an increased incidence of ventricular arrhythmias after the administration of digitalis in the post infarction period, but have been characterized largely as anecdotal, poorly designed, and/or lacking appropriate control groups, and ultimately unpersuasive.$^{19,20}$ In one clinical trial involving 54 patients studied within 48 hours of admission for myocardial infarction, Lown et al. ${ }^{21}$ demonstrated that 48 patients ( $89 \%$ of total) could tolerate the single dose acute intravenous administration of $1.0 \mathrm{mg}$ of acetyl strophanthidin without displaying deleterious conduction disturbances or spontaneous arrhythmias during or up to 30 minutes following drug infusion. Reicansky et al., ${ }^{22}$ in a randomized, double-blinded study, found no differences in the incidences of ventricular arrhythmias during monitoring periods 1 hour before and up to 3 hours after the single dose acute intravenous administration of $0.1 \mathrm{mg} / \mathrm{kg}$ digoxin (18 patients) or saline vehicle (15 patients). Recently, several large retrospective clinical studies have employed multivariate statistical techniques in an attempt to identify potential deleterious effects of chronic digitalis therapy on survival after myocardial infarction. ${ }^{3-7}$ Moss et al. ${ }^{3}$ and Bigger et al. ${ }^{4}$ have reported statistically significant associations between digitalis therapy and mortality at 4 months 
and 1 year after infarction, respectively, in patients with left ventricular failure and ventricular arrhythmias. In contrast to the preceding reports, Ryan et al. ${ }^{5}$ and Madsen et al..$^{6}$ failed to detect a significant, independent association between digitalis therapy and post infarction mortality, although the exclusion of early post infarction deaths in the former study ${ }^{5}$ may have influenced this relationship. Bigger and associates $^{7}$ recently have extended their post infarction patient evaluation throughout a 3-year follow-up period, and have reported the independent association between digitalis therapy and post infarction mortality to be of borderline statistical significance $(0.05<p<0.10)$. Additionally, digitalis therapy recently has been identified as a significant independent predictor of mortality in a patient group consisting of resuscitated victims of cardiac arrest. $^{23}$

Previous experimental studies. Experimental studies conducted to date consistently suggest an enhanced myocardial sensitivity to the toxic effects of digitalis preparations during myocardial ischemia and after myocardial infarction. Early studies demonstrated a significant reduction in the intravenous lethal threshold dosage of tincture of digitalis infused subacutely ${ }^{8}$ or chronically ${ }^{8,11}$ after myocardial infarction in dogs and cats. More recent investigators have demonstrated significant reductions in the intravenous and/or intracoronary arrhythmogenic threshold dosages of digitalis preparations (acetyl strophanthidin or ouabain) infused during myocardial ischemia, ${ }^{y, 10}$ acutely after the reperfusion of an occluded coronary artery, ${ }^{10}$ and at several days, ${ }^{12,13} 1$ month, ${ }^{14}$ and 3 month $s^{15}$ after myocardial infarction in pigs and dogs. However, the clinical relevance of these findings has been questioned because of the consistent use of acute rapid administrations of clearly supratherapeutic and toxic concentrations of the digitalis preparations in assessing myocardial sensitivity in the setting of ischemic myocardial injury. $^{21}$

Present findings: The facilitation of lethal ventricular arrhythmias by therapeutic digoxin in conscious post infarction dogs. In the present study, conscious dogs in the subacute phase of myocardial infarction were used to evaluate the proarrhythmic potential of therapeutically-administered digoxin. The administration of digoxin to post infarction dogs $(0.0125$ $\mathrm{mg} / \mathrm{kg} /$ day intravenously for 5 to 7 consecutive days) yielded trough serum digoxin concentrations of $1.36 \pm 0.17$ and $1.47 \pm 0.19 \mathrm{ng} / \mathrm{ml}$ in animals subjected to repeat programmed stimulation and left circumflex coronary artery intimal injury, respectively. These serum digoxin concentrations compare favorably to the reported clinically therapeutic and nontoxic range of 0.8 to $1.6 \mathrm{ng} / \mathrm{ml}$ serum digoxin. ${ }^{21,25}$

Digoxin-treated post infarction dogs in this study had a significantly greater 24-hour mortality rate after the development of ischemia at a site distant to the area of previous myocardial infarction, as compared to the vehicle-treated post infarction dogs. This enhanced mortality was due to a significantly enhanced vulnerability of the digoxin-treated animals toward the sudden development of ventricular fibrillation in response to the acute ischemic episode. Programmed ventricular stimulation testing before the development of posterolateral ischemia failed to predict this enhanced susceptibility of digoxin-treated post infarction dogs to the development of ischemia-induced lethal arrhythmias, suggesting that the proarrhythmic actions of digitalis may be mediated via an alteration in the electrophysiologic response of the infarcted heart to a new ischemic insult, rather than solely via an effect on the infarcted myocardial substrate. Electrophysiologic measurements conducted during this study detected decreases in myocardial refractoriness and excitability after both digoxin and vehicle treatments, undoubtedly reflecting an evolving electrophysiologic "healing" process after the experimental myocardial infarction. However, the enhanced reduction in myocardial refractoriness observed in the digoxin group, particularly in the infarcted myocardium, as well as the reduction in QT interval (an ECG index for ventricular action potential duration, which in turn is a primary determinant for cellular refractoriness), are consistent with the cellular electrophysiologic actions of digitalis. Through an inhibition in $\mathrm{Na}^{+} / \mathrm{K}^{+}$-ATPase activity, digitalis is thought to cause an elevation in intracellular calcium $\left(\mathrm{Ca}^{2+}{ }_{\text {in }}\right)$ concentration that in turn augments a $\mathrm{Ca}^{2+}{ }_{\text {int }}$-dependent outward $\mathrm{K}^{+}$conductance. This in turn is responsible for the observed acceleration in cardiac cell (phase 3) repolarization, a reduction in cardiac action potential duration, and therefore reduced cellular refractoriness. ${ }^{26}$ Future studies will be required to determine more fully the significance of this enhanced reduction in myocardial refractoriness with regard to the increased susceptibility of digitalis-treated post infarction dogs to ischemiainduced ventricular arrhythmias.

Conclusions. In conscious dogs in the subacute phase of anterior myocardial infarction, the administration of digoxin in a therapeutic dosage regimen resulted in a significantly increased incidence in the development of lethal ventricular arrhythmias in response to ischemia at a site distant to the area of previous myocardial infarction. This enhanced susceptibility of digoxin-treated post infarction dogs to 
the development of ischemia-induced lethal arrhythmias was not predicted by programmed ventricular stimulation testing, and may involve a digoxin-mediated alteration in myocardial refractoriness, particularly in the infarcted myocardium. These experimental findings support clinical observations that digitalis therapy may constitute an independent risk factor contributing significantly to mortality after myocardial infarction.

The authors wish to express their appreciation to John Jarrett and John Larin of the University of Michigan Ligand Assay Laboratory for their assistance in the determination of serum digoxin concentrations, and to Kim Kanitz for her expert preparation of this manuscript.

\section{REFERENCES}

1. Rahimtoola SH, Gunnar RM: Digitalis in acute myocardial infarction: Help or hazard? Ann Intern Med 82:234, 1975.

2. Markus FI: Use of digitalis in acute myocardial infarction. Circulation 62:17, 1980.

3. Moss AJ, Davis H'T, Conard DL, DeCamilla JJ, Odoroff CL: Digitalis-associated cardiac mortality after myocardial infarction. Circulation 64:1150, 1981.

4. Bigger JT, Weld FM, Rolnitzky LM, Ferrick KJ: Is digitalis treatment harmful in the year after acute myocardial infarction? Circulation 64 (suppl TV):83, 1981.

5. Ryan TJ, Bailey KR, McCabe CH, Luk S, Fisher LD, Mock MB, Killip T: The effects of digitalis on survival in high risk patients with coronary artery disease. The Coronary Artery Surgery Study. Circulation 67:735, 1983.

6. Madsen EB, Gilpin E, Henning $\mathrm{H}$, Ahnve S, LeWinter M, Mazur J, Shebetai R, Collins D, Ross J: Prognostic importance of digitalis after acute myocardial infarction. J Am Coll Cardiol 3:681, 1984.

7. Bigger JT, Fleiss JL, Rolnitzky LM, Merab JP, Ferrick KJ: Effect of digitalis treatment on survival after acute myocardial infarction. Am J Cardiol 55:623, 1985.

8. Bellet S, Johnstone CG, Schecter A: Effect of cardiac infarction on the tolerance of dogs to digitalis. Arch Intern Med 54:509, 1934.

9. Kumar R, Hood WB, Joisson J, Gilmour DP, Norman JC, Abelman WH: Experimental myocardial infarction. VI. Efficacy and toxicity of digitalis in acute and healing phase in intact conscious dogs. J Clin Invest 49:358, 1970.

10. Ku DD, Lucchesi BR: Ischemia-induced alterations in cardiac sensitivity to digitalis. Eur J Pharmacol 57:135, 1979.

11. Travell J, Gold $\mathrm{H}$, Modell W: The effect of experimental cardiac infarction on response to digitalis. Arch Intern Med 61:184, 1938 .
12. Hood WB, McCarthy B, Lown B: Myocardial infarction following coronary ligation in dogs. Hemodynamic effects of isoproterenol and acetylstrophanthidin. Circ Res 21:191, 1967.

13. Morris JJ, Taft CV, Whalen RE, McIntosh HD: Digitalis and experimental myocardial infarction. AM HEART J 77:342, 1969.

14. Iesaka Y, Aonuma K, Gosselin AJ, Pinakatt T, Stanford W, Benson J, Sampsell R, Rozansik JJ, Lister WJ: Susceptibility of infarcted canine hearts to digitalis-toxic ventricular tachycardia. .J Am Coll Cardiol 2:45, 1983.

15. Bush LR, Evans RM, Gaul SL: Enhanced sensitivity to the arrhythmogenic effects of digitalis in dogs with previous myocardial infarction. Clin Res 33 (II):172A, 1985.

16. Patterson E, Holland K, Eller BT, Lucchesi BR: Ventricular fibrillation resulting from ischemia at a site remote from previous myocardial infaretion. $\Lambda$ conscious canine model of sudden coronary death. Am J Cardiol 50:1414, 1982.

17. Wilber DJ, Lynch JJ, Montgomery DG, Lucchesi BR: Postinfarction sudden death: Significance of inducible ventricular tachycardia and infarct size in a conscious canine model. AM Heart J 109:8, 1985.

18. Michelson EL, Spear JF, Moore EN: Electrophysiologic and anatomic correlates of sustained ventricular tachyarrhythmias in a model of chronic myocardial infarction. Am J Cardiol 45:583, 1980.

19. Selzer A: The use of digitalis in acute myocardial infarction. Prog Cardiovasc Dis 10:518, 1968.

20. Constant J: Digitalis in myocardial infarction. NY State .J Med 70:650, 1970.

21. Lown B, Klein MD, Barr I, Hagemeijer F, Kosowsky BD, Garrison H: Sensitivity to digitalis drugs in acute myocardial infarction. Am J Cardiol 30:388, 1972.

22. Reicansky I, Conradson T-B, Holmberg S, Ryden L, Waldenstrom A, Wennerblom B: The effect of intravenous digoxin on the occurrence of ventricular tachyarrhythmias in acute myocardial infarction in man. AM HEART J 91:705, 1976

23. Goldstein S, Landis JR, Leighton R, Ritter G, Vasu CM, Wolfe RA, Acheson A, Medendorp SV: Predictive survival models for resuscitated victims of out-of-hospital cardiac arrest with coronary heart disease. Circulation 71:873, 1985.

24. Smith TW: Contribution of quantitative assay techniques to the understanding of the clinical pharmacology of digitalis. Circulation 46:188, 1972.

25. Smith TW, Braunwald E: The management of heart failure. In Braunwald E, editor: Heart disease. Philadelphia, 1980, W.B. Saunders Co, p 509.

26. Hoffman BF: The pharmacology of cardiac glycosides. In Rosen MR, Hoffman BF, editors: Cardiac therapy. Boston, 1983, Martinus Nijhoff Publishers, p 387. 